Urologe 2011 · 50:911-913

DOI 10.1007/s00120-011-2666-8

Online publiziert: 4. August 2011

(c) Springer-Verlag 2011
A. Schroeder ${ }^{1} \cdot$ J. Steffens ${ }^{2}$

${ }^{1}$ Urologische Praxis, Neumünster

${ }^{2}$ Klinik für Urologie und Kinderurologie, zertifiziertes Prostatakarzinom-Zentrum, St.-Antonius-Hospital Eschweiler, Eschweiler

\section{Urologie im Wandel}

\section{Apell der Präsidenten von DGU und BDU: faire Kooperation statt nur mehr Wettbewerb}

Seit einer gescheiterten Agenda 2010 gibt es keine grundlegende Reform im Sozial- und im Gesundheitswesen. Von Perspektiven oder gar einer Strukturreform ist keine Spur.

Versorgungsgesetz, Musterberufsordnung, Vertragsarztrechtänderungsgesetz, Bundesmantelvertrag, GKV-Finanzierungsgesetz und Arzneimittelneuordnungsgesetz stellen alles andere als eine Reform für eine zukunftssichere Gesundheitsversorgung dar. Hier ist Nachhaltigkeit gefordert, wenn man die medizinische Versorgung einer alternden Gesellschaft sicherstellen will: eine Aufgabe für die Politik, aber auch die Leistungserbringer, Kostenträger und Patienten.

Diese Entwicklung hat auch Auswirkungen in unserem urologischen Alltag. Die politischen Rahmenbedingungen ändern sich zunehmend häufiger, sei es durch die Selbstverwaltung oder durch den Gesetzgeber (Bundesmantelvertrag Onkologie, $\ 116 \mathrm{~b}$; Medizinisches Versorgungszentrum). Auferlegte Strukturen und Regulierungen im nationalen Krebsplan (Hämatoonkologen als Leiter onkologischer Zentren) und die Forderung nach Mindestmengen in unterschiedlichen Zentren (Prostatakarzinom-, Beckenboden- und Nierentransplantationszentren) werden mit dem Siegel der Qualitätssicherung versehen.

Diese gesetzlichen Voraussetzungen und kritischen Bestimmungen fordern eine (strategische) Neuausrichtung unseres Fachgebiets. Unsere Urteilskraft, auf die es auch in Wissenschaft, Universität, Klinik und Praxis in erster Linie ankommt, lässt sich nicht durch vermeintlich objektive Verfahren ersetzen. Ob als Arzt in Klinik oder Praxis: Jeder ist gefordert, durch Eigeninitiative Verbünde und Netzwerke zu schaffen, um unser Fach mit seinen unterschiedlichen Schwerpunkten qualifiziert vertreten zu können.

Die Schwerpunkte, aber auch Randgebiete unseres Faches müssen sichtbarer als bisher werden. Sonst gehen urologische Kern- und Teilgebiete verloren. Stärker als bisher müssen wir unsere urologische Dominanz in den Teilgebieten Uroonkologie, rekonstruktive und Kinderurologie, gynäkologische Urologie, Andrologie und Nierentransplantation demonstrieren. Mit der gegenwärtigen Realität in deutschen Kliniken und Praxen hat dies wenig zu tun.

\section{Uroonkologie: Kompetenz zeigen!}

Im vorliegenden Heft setzen sich exponierte Autoren kritisch mit wesentlichen berufspolitischen und fachlichen Aspekten unseres Faches auseinander. Hierzu zählt zunächst die Besinnung auf unser wichtigstes Kerngebiet, die Uroonkologie. $25 \%$ aller soliden Tumoren betreffen den Urogenitaltrakt. Deshalb müssen wir als Urologen und Onkologen Qualifikationen bieten und Therapieentscheidungen selbstbewusster als bisher vertreten.

Die Vorgabe im nationalen Krebsplan, die Krebsbehandlung vermehrt in Zen- tren zu leiten, ist etwas anderes als eine qualifizierte, wohnortnahe und flächendeckende Versorgung. Wir dürfen uns nicht nur als Operateure, sondern auch als Therapeuten für medikamentöse Krebsbehandlung verstehen. In den wöchentlichen Tumorboards muss unsere Leitfunktion für urologische Tumoren durch Präsenz und Kompetenz erkennbar werden. Die medikamentösen Tumorbehandlungsmaßnahmen sollten entweder alleine von Urologen und/oder interdisziplinär, gemeinsam mit den Onkologen umgesetzt werden.

Der erste Beitrag des DKG-Kongresspräsidenten und Urologen Peter Albers fordert ein selbstbewusstes Handeln mit Entwicklung eines eigenständigen Kriterienkataloges zur Behandlung uroonkologischer Patienten. Er ermahnt uns, die Zukunft selbst zu gestalten und dies nicht den politischen Gremien zu überlassen. $\mathrm{Zu}$ diesem Konzept passt das gemeinsam von DGU, BDU und AUO entwickelte Positionspapier, in dem eine spezialisierte operativ-medikamentöse Ausbildung nach dem Facharzt als Schwerpunkt angestrebt wird. Unter dem Aspekt einer künftigen spezialfachärztlichen Versorgung ist dies ein Ansatz, der kritisch zu diskutieren ist.

Von den fünf urologischen Tumoren sind bereits zwei Entitäten Erfolgsmodelle uroonkologischer Leitfunktion: So ist das Prostatakarzinom der einzige Tumor, der unter den fünf DKG-Tumorzentren von Urologen und nicht von Hämatoonkolo- 
gen geleitet wird. Auch die Hodentumorzweitmeinungszentren wurden ohne politischen Druck und in den eigenen Reihen akzeptiert und stellen eine wertvolle Entscheidungshilfe im klinischen Alltag dar. Gschwend u. Schrader gehen auf die Entwicklungsmöglichkeiten dieser Versorgungsformen ein.

Der onkologische Versorgungsbedarf muss sektorenübergreifend betrachtet werden. Michel beschreibt die Möglichkeiten einer engen Verzahnung zwischen Klinik und Praxis in der Versorgungsforschung. In diesem Aufsatz spornt er niedergelassene und klinisch tätige Kollegen an, sich mit diesem bisher stiefmütterlich behandelten Gebiet der Versorgungsforschung intensiver zu beschäftigen, um die uroonkologische Zukunft sektorübergreifend zu gestalten.

\section{Roboterchirurgie: Hype schon vorbei?}

Kaum eine Technologie hat die Urologie in den letzten 10 Jahren so revolutioniert und polarisiert wie das Robotersystem Da Vinci. Trotz faszinierender Operationstechnik ist ein Nutzen für die Patienten bisher nicht belegt worden. Onkologisches und funktionelles Outcome kann aufgrund der noch jungen Technologie und verständlicherweise fehlender Langzeiterfahrungen noch nicht endgültig beurteilt werden. In den Händen des erfahrenen High-volume-Operateurs ist diese Technik nicht schlechter als die bisher verwendeten. Siemer u. Stöckle beschreiben kritisch die Zukunft dieser urologischen Innovation und machen deutlich, dass nur High-volume-Operateure mit guten Operationsergebnissen und niedrigen Komplikationsraten die betriebswirtschaftlichen Unkosten einschränken können. Diese Technik wird nach einer Phase der Desillusionierung nicht flächendeckend, sondern nur von großen Zentren erfolgreich betrieben werden.

\section{Forschung sichert Zukunft}

Die Zukunftssicherung der Urologie erfolgt auch durch Grundlagenforschung. Mit systematischer Nachwuchsförderung kann einem Mangel an Fachkräften entgegengewirkt werden. Die DGU versteht es seit 3 Jahren, das Potential, das in der Unterstützung unserer Mediziner liegt, über eine umfassende neue Initiative auszuschöpfen. In diesem Jahr wird mit der zweiten Vergabe der Eisenberger-Forschungsstipendien an urologische Assistenzärzte die zukunftsorientierte, qualifizierte Weiterbildung in der urologischen Forschung gefördert und gleichzeitig ein weiterer Anreiz zur Entscheidung für unser Fachgebiet geschaffen. Junker u. Wullich demonstrieren eindrucksvoll die ersten Früchte einer translationalen Forschung. An einigen urologischen Universitätskliniken konnten definierte Forschungsvorhaben etabliert und interdisziplinäre Arbeitsgruppen aufgebaut werden, um die urologische Forschung nachhaltig zu stimulieren.

In der aktuellen Debatte über die Kosten des deutschen Gesundheitssystems wird gefordert, medizinische Innovationen besser auf ihren Nutzen zu überprüfen. Politische Spitzenverbände stellen konsequent folgende Fragen: Nützt das Verfahren? Wem nützt es? Wann soll es eingesetzt werden? Welche Qualitätsanforderungen müssen unbedingt beachtet werden, damit das Behandlungsziel erreicht werden kann? Vergleichende klinische Studien werden gefordert, um Nutzen und Qualität der Behandlungsmaßnahmen transparent $\mathrm{zu}$ machen. Damit wären auch die Voraussetzungen für einen fairen Wettbewerb und wirklich nützliche Innovationen geschaffen. Fragwürdige Neuerungen könnten frühzeitig erkannt und eliminiert werden. Das käme den Patienten zu Gute und würde unnötige Ausgaben im Gesundheitswesen vermeiden. Vor diesem Hintergrund bietet die geplante PREFERE-Studie eine Chance für uns Urologen.

Schmedders et al. weisen auf die Evidenzprobleme bei der Behandlung des Niedrigrisikoprostatakarzinoms hin. Stöckle geht in seinem konstruktiven Kommentar auf die Hintergründe, Vorgaben und Chancen dieser interdisziplinären und intersektoralen Studie ein. Aufgrund der hohen geforderten Fallzahl und der nicht unproblematischen Randomisierung durch einen mit den Spielregeln der evidenzbasierten Medizin wenig vertrauten deutschen Patienten wird dieses Projekt zwar kritisch bewertet, je- doch als notwendige Anstrengung gesehen, um das Ansehen unseres Faches im Interesse der uns anvertrauten Patienten zu stärken. Hier kommt auf die niedergelassenen Urologen die wesentliche Aufgabe der Aufklärung zu.

\section{Urologische Ausbildung muss besser werden}

Im europäischen Kontext ist die gegenwärtige Aus- und Weiterbildung zum Urologen nicht mehr zeitgemäß. Im Vergleich zu anderen Ländern gibt es Defizite, die von Müller u. Strunk klar formuliert werden. Möglichkeiten und Maßnahmen einer Qualitätsverbesserung werden dargestellt. Die großen Diskrepanzen innerhalb Europas könnten durch ein gemeinsames Curriculum für die urologische Ausbildung ausgeglichen werden.

Die Zusatzweiterbildung Andrologie hat sich nach Ansicht von Kliesch u. Weidner mittlerweile etabliert und die Andrologie ist zu einem eigenständigen Versorgungsgebiet geworden. Die hohe Anzahl von fast 1500 Androloginnen/Andrologen ist einer Übergangsregelung geschuldet, die Fachärzte für Urologie aus Klinik und Praxis nutzen konnten, um die Zusatzbezeichnung Andrologie zu führen. Bei nur einem Zehntel der Ausbildungsbefugten ist die Sicherstellung der Andrologie in Zukunft nur bei künftig ausreichend vorhandenen Weiterbildungsstellen gewährleistet, so die Autoren Kliesch u. Weidner.

Das moderne Berufsbild und die Perspektive des Urologen in der Praxis werden von Dohr treffend beschrieben. Er zeigt auf, dass die Bewertung der Attraktivität des Arztberufs keineswegs nur eine Frage des Geldes ist, sondern ganz wesentlich auch von anderen Faktoren (,work life balance") bestimmt wird. Er fordert verlässliche Rahmenbedingungen, Planungssicherheit für die Praxen, jedoch auch Leidenschaft und Begeisterung vom Praxiseigentümer.

Die bestehenden fachlichen und berufspolitischen Herausforderungen unseres Faches sind vielschichtig und nicht überall in gleicher Weise von jedem zu leisten. Wichtig ist die Schaffung von Berufsausübungsgemeinschaften (BAG), Netzwerken und Verbünden, um möglichst viele Teilgebiete unseres faszinie- 
renden Faches in Kooperationen kompetent abzudecken. Dies erfordert Engagement, Flexibilität und Teamfähigkeit zum Aufbau von neuen Strukturen und Prozessen und stellt eine vielversprechende, spannende Aufgabe in der urologischen Klinik und Praxis dar.

So kann die Zukunftsfähigkeit unseres Faches gesichert werden. Urologische Versorgung wird in einer alternden Gesellschaft mehr denn je gebraucht.

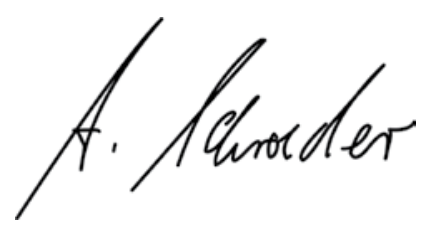

Dr. A. Schroeder, BDU-Präsident

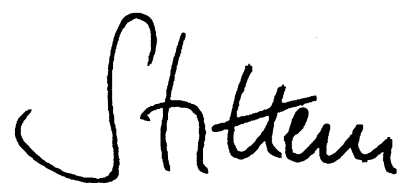

Prof. Dr. J. Steffens, DGU-Präsident

\section{Korrespondenzadresse}

Dr. A. Schroeder

Urologische Praxis,

Haart 87-89, 24534 Neumünster

drschroeder@bdu-praesident.de

\section{Premiere für DGU-Kongress-App}

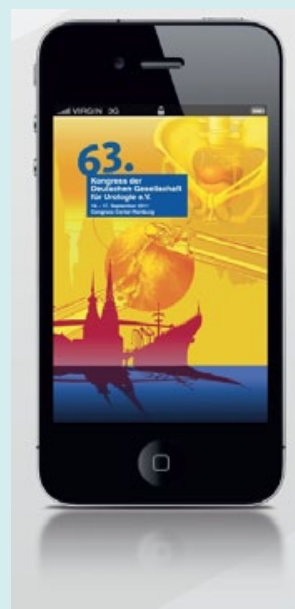

Die zeitgemäßen Möglichkeiten moderner Kommunikationsgeräte machen sich nun auch die Urologen zunutze: Zum 63. Kongress der Deutschen Gesellschaft für Urologie e.V. (DGU) vom 14. bis 17. September 2011 in Hamburg wird es erstmals eine spezielle Applikation für

Smartphones geben. Die kostenlose „DGUKongress-App" enthält nicht nur das umfangreiche Hauptprogramm der Jahrestagung in digitaler Form, sondern bietet auch eine Vielzahl weiterer nützlicher Funktionen. Ab Mitte August wird sie zum Download zur Verfügung stehen.

„Eine der wichtigsten Funktionen der App ist der Programmplaner: Durch einfaches Anklicken von Veranstaltungen, Vorträgen, Präsentationen, Ausstellern oder anderen Angeboten aus dem Kongressprogramm stellt der Anwender seinen individuellen Kongressplan zusammen", sagt DGU- und Kongress-Präsident Prof. Dr. Joachim Steffens. Für jeden Termin seien alle entsprechenden Informationen, wie etwa zu Fachgebieten, Referenten und Unternehmen, abrufbar und auch die räumliche Orientierung im $\mathrm{CCH}$ werde unterstützt und erleichtert. Terminänderungen werden per Kurznachricht mitgeteilt (Push Notification) und im individuellen Kongressplan automatisch aktualisiert, wenn das Smartphone online ist.

Zu den Funktionen der App gehört auch die Übermittlung von Videostreams. Die drei Plena des Kongresses werden mitgeschnitten und etwa eine Stunde nach Ende der Veranstaltungen online gestellt. Für die Nutzung dieses Dienstes ist jedoch ein Passwort notwendig, welches nur registrierte Kongressteilnehmer erhalten.

„Der Einsatz der DGU-Kongress-App ist eine Premiere für uns. Deshalb belassen wir es zunächst bei den üblichen Hauptfunktionen und reizen nicht alles aus, was heute technisch möglich wäre. So bietet der mobile Kongressplan für das Smartphone eine Fülle nützlicher Funktionen, ohne den Anwender zu überfordern", sagt DGU-Pressesprecherin Prof. Dr. Sabine Kliesch.

Die „DGU-Kongress-App“ wird es ab 14.

August 2011 für folgende derzeit gängige
Smartphones und Mobilgeräte bzw. Betriebssysteme geben:

- iPhone/iPad von Apple mit Betriebssystem iOS 4.0 oder neuer (Download über iTunes App Store, Suchbegriff: „DGU“)

- BlackBerry mit Betriebssystem ab Version 4.5 (Download über http://dgu.mobileeventguide.de/blackberry)

- alle Smartphones mit Android-Betriebssystem ab Version 1.6 (Download über Android Market, Suchbegriff: „DGU“)

- des Weiteren finden Sie unter http:// dgu.mobileeventguide.de eine für alle anderen internetfähigen Smartphones optimierte mobile Website

Weitere Informationen: DGU-Kongress-Pressestelle Bettina-Cathrin Wahlers Sabine Martina Glimm redaktion@bettina-wahlers.de 